\title{
IL-17 contributed to the neuropathic pain following peripheral nerve injury by promoting astrocyte proliferation and secretion of proinflammatory cytokines
}

\author{
CAIXIA SUN ${ }^{1,2}$, JIN ZHANG $^{1}$, LI CHEN $^{1}$, TANGHUA LIU ${ }^{1}$, GAOBING XU $^{3}$, \\ CHUNYE LI ${ }^{1}$, WEN YUAN ${ }^{1}$, HUAXI XU ${ }^{2}$ and ZHAOLIANG SU ${ }^{2}$ \\ ${ }^{1}$ Department of Anesthesiology, The Affiliated Hospital of Jiangsu University, Zhenjiang, Jiangsu 212001; \\ ${ }^{2}$ Department of Immunology, School of Medicine, Jiangsu University, Zhenjiang, Jiangsu 212013; \\ ${ }^{3}$ Central Laboratory, The Affiliated People's Hospital of Jiangsu University, \\ Zhenjiang, Jiangsu 212001, P.R. China
}

Received September 30, 2015; Accepted October 11, 2016

DOI: $10.3892 / \mathrm{mmr} .2016 .6018$

\begin{abstract}
Central neuroinflammation is important in the pathophysiological processes of neuropathic pain following peripheral nerve injury. Recently, interleukin-17 (IL-17) has been detected in different inflammatory conditions of the central nervous system and contributes to neuropathic pain associated with multiple sclerosis, experimental autoimmune encephalomyelitis. The present study, based on the rat model of spinal nerve ligation, analyzed the infiltration of cluster of differentiation (CD) $4^{+} \mathrm{T}$ cells and the expression of IL-17 in the spinal cord during the maintenance phase of neuropathic pain, and investigated central inflammatory reaction and astrocyte activation. The results demonstrated that the infiltrated $\mathrm{CD} 4^{+}$ $\mathrm{T}$ cells in the spinal cord increased in the rat model of spinal nerve ligation, and immunofluorescence staining demonstrated that the $\mathrm{CD}^{+} / \mathrm{IL}-17^{+}$cells were located at superficial laminae of spinal dorsal horn. This was accompanied by significant upregulation of IL-17. Furthermore, the mRNA expression levels of IL-1 $\beta$ and IL- 6 were also significantly enhanced in model rats compared with the sham and control groups in the spinal dorsal horn. In vitro, the proliferation ability and secretion of proinflammatory cytokines notably increased in the IL-17-stimulated astrocytes. Results from the present study indicate that IL-17 may contribute to neuropathic pain by promoting the proliferation of astrocytes and secretion of proinflammatory cytokines in spinal nerve ligation-induced neuropathic pain.
\end{abstract}

Correspondence to: Dr Zhaoliang Su, Department of Immunology, School of Medicine, Jiangsu University, 301 Xuefu Road, Zhenjiang, Jiangsu 212013, P.R. China

E-mail: szl30@126.com

Key words: neuropathic pain, IL-17, model of spinal nerve ligation, rat

\section{Introduction}

Neuropathic pain, defined as pain due to a lesion or disease of the somatosensory system (1), is one of the most severe forms of chronic pain. It may be induced by nerve trauma, infection, metabolic disease, ischemia, radiotherapy or chemotherapy. It is often refractory to current treatments, partially due to incomplete understanding of the underlying mechanisms. Nerve injury or disease may result in chronic central neuroinflammation in the dorsal horn of the spinal cord and along the pain pathways to the thalamus and the parietal cortex $(2,3)$. Central neuroinflammation modulates synaptic plasticity and facilitates nociceptive signal transmission, which eventually develops into chronic pain (4,5). Extensive evidence now indicates glial cells are critical in central neuroinflammation following nerve injury (6-8). The activation of astrocytes may explain the long-lasting behavioral hypersensitivity $(9,10)$.

Astrocytes are the most abundant glial cells in the central nervous system (CNS), and were historically regarded as support cells. However, previous data indicates that astrocytes have multiple active roles in acute and chronic neuronal diseases, including seizure, stroke, and ischemia $(9,11,12)$. Furthermore, accumulating evidence has demonstrated that astrocytes are important for maintenance of neuropathic pain $(7,9,10)$. In all models of neuropathic pain, such as rhizotomy (8), chronic constriction injury (13) and spinal nerve ligation (14), proliferation and activation of astrocytes has been demonstrated, and inhibiting astrocytes in the spinal cord was demonstrated to reduce neuropathic pain $(13,14)$. However, the activation of astrocytes and how they mediate neuropathic pain requires further elucidation.

Interleukin-17A (IL-17) is the first member of a family of cytokines, designated IL-17A-F, which are predominantly produced by activated cluster of differentiation (CD) $4^{+} \mathrm{T}$ cells. IL-17 has potent proinflammatory properties and it is involved in modulating the immune response in inflammatory disorders (15). Furthermore, previous studies also demonstrated that IL-17 is involved in pain. IL-17 or IL- $17^{+} \mathrm{T}$ cells have been observed in patients suffering from arthritic pain (16), in 
the sciatic nerve $(17,18)$, or in optic nerve injury (19) induced animal models of neuropathic pain. Pain-associated behavior has been demonstrated to be reduced in IL-17 knockout (KO) mice in inflammatory models with complete Freund's adjuvant (CFA) injection into a plantar or the sciatic nerve (20) and in peripheral nerve injured models $(2,18)$. These previous studies suggest peripheral IL-17 exerts an effect in inflammatory and neuropathic pain. Intrathecal injection of recombinant IL-17 promotes thermal hyperalgesia of normal mice (20), which demonstrates that central IL-17 is a key factor in inflammatory pain. The role of IL-17 in the CNS in models of neuropathic pain was suggested by $\mathrm{CD}^{+} \mathrm{T}$ cell infiltration (21), and its elevated concentration in the spinal cord following nerve injury (22). In addition, inflammatory disorders that are associated with IL-17 and Th17 cells, including multiple sclerosis (MS) or allergic encephalomyelitis, often result in the development of neuropathic pain (23). However, the specific role of central nerve system IL-17 in nerve injury-induced neuropathic pain has not been extensively investigated.

The aim of the present study was to determine whether IL-17 was highly expressed in the spinal cord and investigate which cell produced it during the maintenance phase of neuropathic pain. The association between IL-17 and astrocytes was also investigated by analyzing the biological characteristics of IL-17-stimulated astrocytes in cell proliferation and secretion of proinflammatory cytokines in vitro.

\section{Materials and methods}

Animals. A total of 116 male Sprague-Dawley rats (age, 8-10 weeks; weight, 180-250 g) were obtained from the Experimental Animal Center of Jiangsu University (Zhenjiang, China) and acclimatized to the environment for at least 1 week prior to use in the experiments. The environment was maintained at a constant room temperature and humidity level $\left(22^{\circ} \mathrm{C}\right.$; relative humidity, $\left.40-60 \%\right)$. All rats were housed with access to food and water ad libitum and maintained in a 12:12 h light/dark cycle. The well-being of the animals was monitored daily. All animal experiments were approved by the Animal Care and Ethics Committee of Jiangsu University.

Ligation of L5 and L6 spinal nerves. Surgery was performed according to the method described previously (24). Briefly, rats were anesthetized with pentobarbital sodium $(50 \mathrm{mg} / \mathrm{kg}$ i.p.) and a longitudinal incision overlying the lumbar (L)4-sacral (S)1 section was made and the left muscle tissue was separated. The L6 transverse process and L6/S1 posterior interarticular process were exposed and transected. The L5 and L6 spinal nerve was carefully exposed and the distal nerve of L5 and L6 were gently separated, firmly ligated with 5-0 silk suture and transected. The wound was irrigated with sterile saline and inspected for homeostasis, and closed. Sham surgery was performed identically, however, the nerves were not ligated. The animals were allowed to emerge from anesthesia in the warming place. The naive group did not undergo an operation.

Testing mechanical sensitivity. The animals were habituated to the behavioral testing apparatus for $30 \mathrm{~min}$ to $1 \mathrm{~h}$ prior to testing. The testing environment was kept quiet and all tests were conducted in the morning between 8:30 and 11:30.
Mechanical sensitivity of the rats' left feet was measured at $24 \mathrm{~h}$ prior to (baseline value) and at days 1, 3, 5, 7, 10 and 14 after surgery. The midplantar surface of the rats' left hind paws was stimulated using one of a series of eight von Frey filaments (Stoelting Co., Wood Dale, IL, USA) ranging from 0.4 to $15.0 \mathrm{~g}$ ( $\log$ force $3.61,3.84,4.08,4.31,4.56,4.74,4.93$, 5.18) using the Up-Down methods (25). The von Frey filament was presented perpendicular to the plantar surface with sufficient force to result in slight buckling against the paw for $\leq 8$ sec. Paw withdrawal was considered a positive response. The $50 \%$ mechanical withdrawal threshold (50\% MWT) was calculated. If the value of 50\% MWT exceeded $4.0 \mathrm{~g}$ at day 7 post-surgery, the animal model was considered a failed preparation and excluded from the experiment. A total of 8 rats were included in each group. The person performing the behavioral tests was blinded to the experimental groups.

Reverse transcription-quantitative polymerase chain reaction $(R T-q P C R)$. Rats were deeply anesthetized by inhalation of isoflurane $(4 \%)$ in $100 \% \mathrm{O}_{2}$ and intracardially perfused with $0.9 \%$ saline (between 200 and $250 \mathrm{ml}$ per rat at $4^{\circ} \mathrm{C}$ ), and the spinal cords were subsequently harvested. The lumbar spinal dorsal horns from the L5 to L6 ipsilateral to injury were dissected and rapidly frozen. The tissues were homogenized and total RNA was extracted with TRIzol reagent (Invitrogen; Thermo Fisher Scientific, Inc., Waltham, MA, USA). In the in vitro experiment, total RNA was extracted from astrocytes with $100 \mathrm{ng} / \mathrm{ml}$ IL-17 (R\&D Systems, Inc., Minneapolis, MN, USA) treatment for three days or without. Interleukin-6 (IL-6), interleukin-1 $\beta$ (IL-1 $\beta$ ), tumor necrosis factor- $\alpha$ (TNF- $\alpha)$, and/or IL-17 mRNA were measured by RT-qPCR. RT-qPCR was performed with the Verso 1-step RT-qPCR kit, SYBR Green, low ROX (Invitrogen; Thermo Fisher Scientific, Inc.) according to the manufacturer's protocol and the thermocycling conditions were as follows: $5 \mathrm{~min}$ at $94^{\circ} \mathrm{C}$; followed by 35 cycles of $10 \mathrm{sec}$ at $94^{\circ} \mathrm{C}, 5 \mathrm{sec}$ at $50^{\circ} \mathrm{C}$, and $10 \mathrm{sec}$ at $72^{\circ} \mathrm{C}$. Relative expression was calculated with normalization to $\beta$-actin values (26). The primers used are presented in Table I.

Immunohistochemistry staining. Rats were deeply anesthetized by inhalation of isoflurane (4\%) in $100 \% \mathrm{O}_{2}$ and intracardially perfused with $0.9 \%$ saline (between 200 and $250 \mathrm{ml}$ per rat at $4^{\circ} \mathrm{C}$ ), followed by $4 \%$ formaldehyde in $0.1 \mathrm{M}$ phosphate-buffered saline (PBS; between 300 and $350 \mathrm{ml}$ per rat). The L5 segment of lumbar spinal cord was harvested, postfixed overnight in $4 \%$ formaldehyde and then dehydrated in 30\% sucrose for at least $72 \mathrm{~h}$ at $4^{\circ} \mathrm{C}$. Tissue was then freeze-mounted in optimal cutting temperature embedding medium (Sakura Finetek USA, Inc., Torrance, CA, USA) on cork blocks for cryostat sectioning. Spinal cords were sectioned transversely at a thickness of $20 \mu \mathrm{m}$. The sections were washed three times with PBS. Sections were then blocked for $1 \mathrm{~h}$ at $37^{\circ} \mathrm{C}$ with blocking solution [PBS containing $5 \%$ bovine serum albumin (BSA; Shanghai Universal Biotech Company, Shanghai, China) and $0.25 \%$ Triton X-100] followed by incubation with the primary antibody diluted in PBS containing $1 \%$ BSA at $4^{\circ} \mathrm{C}$ overnight. Primary antibodies used were as follows: Monoclonal purified goat anti-rat CD4 molecular complex antibody (1:100; Santa Cruz Biotechnology, Inc., Dallas, TX, 
Table I. Primers used in the present study.

\begin{tabular}{llc}
\hline \multirow{2}{*}{ Gene } & \multicolumn{1}{c}{ Sequence (5'-3') } & $\begin{array}{c}\text { Length } \\
(\mathrm{bp})\end{array}$ \\
\hline IL-6 & U: CCGGAGAGGAGACTTCACAG & 161 \\
& L: ACAGTGCATCATCG CTGTTC & \\
IL-1 $\beta$ & U: AGGCTTCCTTGTGCAAGTGT & 230 \\
& L: TGAGTGACACTGCCTTC CTG & \\
TNF- $\alpha$ & U: AGTCCGGGCAG GTCTACTTT & 230 \\
& L: CGTGTGTTTCTGAGCATCGT & \\
IL-17 & U: ACAGTGAAGGCAGCGGTACT & 172 \\
$\beta$-actin & L: GAGTCCAGGGTGAAGTGGAA & \\
& U: CACGAAACTACCTTCAACTCC & 265 \\
& L: CATACTCCTGCTTGCTGATC &
\end{tabular}

$\mathrm{U}$, upper; L, lower; IL, interleukin; TNF- $\alpha$, tumor necrosis factor- $\alpha$.

USA; cat. no. sc-1140) for the detection of infiltrating CD4 ${ }^{+}$ cells; rabbit anti-rat IL-17 (1:100; Santa Cruz Biotechnology, Inc.; cat. no. sc-7927); and mouse anti-rat glial fibrillary acidic protein (GFAP, 1:1,000; Santa Cruz Biotechnology, Inc.; cat. no. sc-135921) for the detection of astrocytes. The sections were then washed five times with PBS and incubated with the appropriate secondary antibody for $1 \mathrm{~h}$ at room temperature. Secondary antibodies used were as follows: Fluorescein isothiocyanate (FITC)-conjugated donkey anti-goat (1:100; Santa Cruz Biotechnology, Inc.; cat. no. sc-2042), phycoerythrin (PE)-conjugated donkey anti-rabbit (1:100; Santa Cruz Biotechnology, Inc.; cat. no. sc-3745), and PE-conjugated goat anti-mouse (1:250; Invitrogen; Thermo Fisher Scientific, Inc.; cat. no. M30004). Following washing with PBS, the sections were stained with Hoechst 33258 at $37^{\circ} \mathrm{C}$ for $5 \mathrm{~min}$ and the cells were examined with a fluorescence microscope (Olympus BX51; Olympus Corporation, Tokyo, Japan).

Fluorescence-activated cell sorting (FACS) was used to detect the infiltrated $C D 4^{+} T$ cells in spinal cord. The ipsilateral (relative to injury) lumbar dorsal horns of L5-L6 spinal cord were harvested as described above. Mononuclear cells from the spinal cord tissue were obtained with discontinuous Percoll gradients method following a previously described method (27). Due to the limited numbers of mononuclear cells from a single animal, each FACS sample was prepared from three spinal cords pooled together. The collected mononuclear cells was suspended in $100 \mu \mathrm{l}$ PBS containing anti-rat CD3-FITC (1:100; eBioscience, Inc., San Diego, CA, USA; cat. no. 11-0030) and anti-rat CD4-PE (1:100; eBioscience, Inc.; cat. no. 12-0040). Cells were incubated at $4^{\circ} \mathrm{C}$ for $30 \mathrm{~min}$. Following washing twice with PBS, all stained cells were resuspended in $4 \%$ formaldehyde/PBS at $4^{\circ} \mathrm{C}$ overnight. Data were acquired by flow cytometry (BD Biosciences, Franklin Lakes, NJ, USA) and analyzed using CellQuest software (version 2.8; BD Biosciences).

ELISA for serum IL-17. Serum IL-17 was measured by an ELISA kit (Rat IL-17A Platinum ELISA; eBioscience, Inc.) following the manufacturer's protocols. All samples were measured in triplicate, and the mean concentration was calculated from the standard curve.

Astrocyte culture and cell proliferation assay. As previously described (28), astrocyte cultures were prepared from 1-2 day-old postnatal rats. Briefly, in a sterile environment, spinal cords were dissected and cell suspensions were prepared. These were subsequently transferred to culture flasks and incubated at $37{ }^{\circ} \mathrm{C}$ in a $\mathrm{CO}_{2}$ balanced incubator. After 7 days, the flask was agitated at $200 \mathrm{rpm}$ at $37^{\circ} \mathrm{C}$ overnight to remove oligodendrocytes, neurons, and microglial cells. Following three passages, the astrocytes were divided into IL-17-stimulated and naive groups. Briefly, $1 \times 10^{3}$ cells/well were seeded into 96 -well plates in Dulbecco's modified Eagle's medium (Gibco; Thermo Fisher Scientific, Inc.) and following cell adherence to the plate, IL-17 was added to the wells at concentration of 10,50 or $100 \mathrm{ng} / \mathrm{ml}$, and incubated at $37^{\circ} \mathrm{C}$ for $24-72 \mathrm{~h}$. Subsequently, $10 \mu \mathrm{l}(5 \mathrm{mg} / \mathrm{ml}) \mathrm{MTT}$ was added to each well and the plate was further incubated for $4 \mathrm{~h}$ to deoxidize MTT under light-blocking conditions. Following removal of the MTT dye solution, the cells were treated with $100 \mu \mathrm{l}$ DMSO and the absorbance at a wavelength of $490 \mathrm{~nm}$ was measured using the ELx800 UV microplate reader (BioTek Instruments, Inc., Winooski, VT, USA). Cells from the naive group were not stimulated by IL-17.

Statistical analysis. All data were expressed as the mean \pm standard deviation. Figures and statistical analyses were made with GraphPad Prism 5.0 (GraphPad Software, Inc., La Jolla, CA, USA). For comparison of mechanical sensitivity, spinal inflammatory cytokines, infiltrated-cellular phenotypes in the spinal cord and the MTT assay, appropriate two-way analysis of variance was performed followed by Bonferroni's multiple comparison post-hoc test. Immunohistochemistry data were analyzed by t-test or one-way analysis of variance. Inflammatory cytokines data in vitro were evaluated using t-test. $\mathrm{P}<0.05$ was considered to indicate a statistically significant difference.

\section{Results}

Behavioral mechanical sensitivity of rats following L5/L6 spinal nerve ligation. The mechanical sensitivity (indicated by the $50 \%$ threshold force for paw withdrawals) was measured in the injured ipsilateral hind paws prior to (baseline values) and up to 2 weeks after surgery. Baseline values were not different between the groups. Following SNL, rats developed pain hypersensitivity at day 3 up to day 14 compared with the other two groups. Furthermore, no significant differences were observed between the sham group and the control group at any time point (Fig. 1).

$C D 4^{+}$Th17 cell infiltration was observed in the ipsilateral dorsal horn following L5-L6 spinal cord ligation. Following spinal nerve ligation, IL-17 mRNA increased significantly in the ipsilateral dorsal horn of L5-L6 spinal cord at day 7 $(\mathrm{P}<0.05)$. No differences were detected between the sham and control group at any time (Fig. 2A). Serum IL-17 in naive, sham-operated, and SNL rats at days 7 and 14 


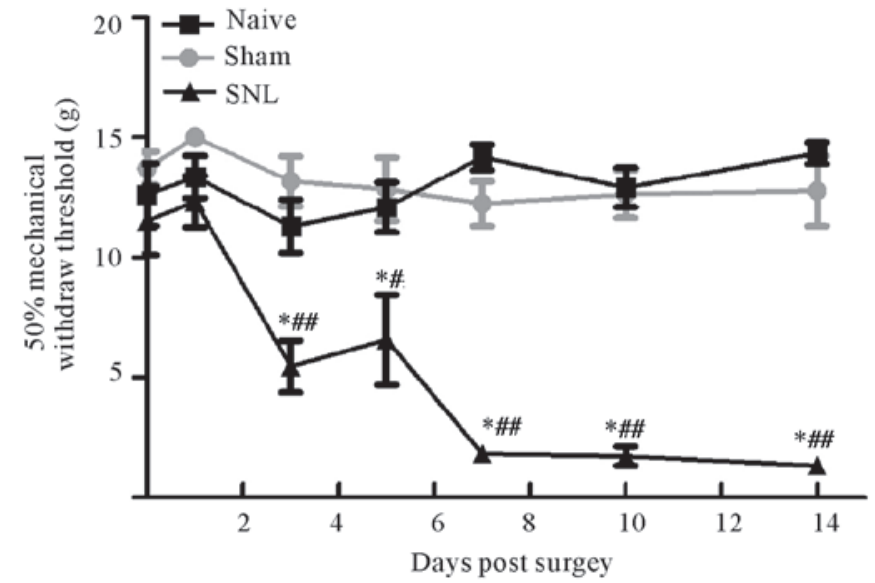

Figure 1. Behavioral responses for mechanical sensitivity of rats induced by unilateral ligation of the L5 and L6 spinal nerves compared with sham-operated or naive group. Mechanical sensitivity was presented as the $50 \%$ withdrawal threshold for each animal (mean \pm standard error of the mean, $\mathrm{n}=8$ ). ${ }^{*} \mathrm{P}<0.001$ vs. the sham-operated rats; ${ }^{\#} \mathrm{P}<0.01,{ }^{\# \#} \mathrm{P}<0.001$ vs. the naive rats, as determined by two-way analysis of variance, followed by Bonferroni's post-hoc test. SNL, spinal nerve ligation; L, lumbar.

post-surgery was analyzed by ELISA to determine whether the changes of IL-17 in spinal cord was associated with the changes in the peripheral serum. Though spinal nerve ligation induced a moderate increase in IL-17 expression, no differences were determined amongst the three groups at any time point (Fig. 2B). Furthermore, IL-17 localization was detected in the spinal cord at day 7 after surgery. As IL-17 is preferentially expressed by $\mathrm{CD}^{+} \mathrm{T}$ cells, double immunofluorescence labeling was used to demonstrate that IL-17 immunoreactivity was colocalized with CD4 in the ipsilateral dorsal horn, and the immunostaining density of IL-17 and CD4 markedly increased when compared with the sham-operated rats (Fig. 2C).

To further confirm IL-17 was produced by spinal cord-infiltrating $\mathrm{CD}^{+}{ }^{+} \mathrm{T}$ cells, $\mathrm{CD} 4^{+} \mathrm{T}$ cells were detected in the lumbar spinal cord using FACS at days 7 and 14 post-surgery. SNL induced a significant increase in the infiltration of $\mathrm{CD}^{+}$ $\mathrm{T}$ cells into the ipsilateral side of lumber spinal cord at day 7 $(\mathrm{P}<0.001)$, while no marked changes were observed at day 14 compared with the naive and sham groups (Fig. 2D).

Astrocyte activation was observed in spinaldorsal horn accompanying proinflammatory cytokine increases following SNL. SNL was demonstrated to induce marked expression of GFAP at the ipsilateral side compared with either the contralateral spinal cord or either side of the sham group. The level of GFAP in the ipsilateral side was consistent with the contralateral side in the sham group. GFAP activation was predominantly localized to the superficial laminae of the lumbar spinal dorsal horn. The immunoreactivity of GFAP notably increased compared with the sham-operated rats (Fig. 3A). Furthermore, the qPCR indicated significant increases in the expression levels of IL-6 and IL-1 $\beta$ in the ipsilateral spinal dorsal horn following SNL at day $7(\mathrm{P}<0.05$ and $\mathrm{P}<0.01)$ and IL-6 remained at high levels at day $14(\mathrm{P}<0.05$; Fig. 3B and $\mathrm{C})$. No change in expression of TNF- $\alpha$ was demonstrated at days 7 and 14 in all groups (data not shown).
IL-17 promoted the proliferation of astrocytes and the expression of proinflammatory cytokines in vitro. To investigate the effect of IL-17 on astrocytes, 10, 50 or $100 \mathrm{ng} / \mathrm{ml}$ IL-17 was used to stimulate astrocytes in vitro. The results of the MTT assay demonstrated that astrocytes proliferated successively over time, but not significantly higher than that at $24 \mathrm{~h}$ in each group. IL-17 induced significant astrocyte proliferation at $48 \mathrm{~h}$ compared with the naive group $(\mathrm{P}<0.05)$. However, at $72 \mathrm{~h}$, only $100 \mathrm{ng} / \mathrm{ml} \mathrm{IL-17}$ significantly promoted proliferation $(\mathrm{P}<0.01$; Fig. 4A). Thus, the expression levels of IL-1 $\beta$, IL- 6 and TNF- $\alpha$ mRNA at $72 \mathrm{~h}$ between the group with $100 \mathrm{ng} / \mathrm{ml} \mathrm{IL-17} \mathrm{stimu-}$ lation and the naive group were investigated. Treatment with $100 \mathrm{ng} / \mathrm{ml}$ IL-17 elevated IL-1 $\beta$ and IL-6 mRNA expression levels (Fig. 4B), but not TNF- $\alpha$ (data not shown). These results demonstrate that IL-17 enhanced astrocytic activity.

\section{Discussion}

Damage to a peripheral nerve results in activity from microglial and astrocytic cells, in addition to infiltration of macrophages and T cells in the CNS. These non-neuronal cells can sensitize neurons in nociceptive pathways by release of pronociceptive factors, including cytokines and chemokines, which is an important factor underlying the pathogenesis of neuropathic pain (29). Nerve injury induced behavioral hypersensitivity can be divided into at least two phases, an initiation phase (from day 0 to day 5) and a maintenance phase (after day 5) based on the pattern of nociceptive behaviors and the animals' responses to selective treatment with therapeutic agents. The spinal cord is an important gateway through which peripheral pain signals are transmitted to the brain. The results of the present study determined proinflammatory IL-17 contributed to the persistence of tactile hypersensitivity following nerve injury.

IL-17 is typically produced by immune cells, including Th17, $\gamma \delta \mathrm{T}$ cells and natural killer T cells, $\mathrm{CD}^{+} \mathrm{T}$ cells (30). The present study determined that IL-17 increases in the ipsilateral spinal cord dorsal horn at day 7 post-SNL and observed IL-17 is located in $\mathrm{CD}^{+}$cells via double immunostaining. Previous studies have detected $\mathrm{CD}^{+}$cells in the lumbar spinal cord or dorsal root ganglia in animal models of neuropathic pain $(21,31)$ and demonstrated these CD4-expressing cells were predominantly CD4 ${ }^{+} \mathrm{T}$ cells (21). The current study confirmed $\mathrm{CD}^{+} \mathrm{T}$ cell infiltration in the spinal cord at day 7 post-nerve injury. The temporal profile of $\mathrm{CD}^{+} \mathrm{T}$ cells is consistent with IL-17 expression in the spinal cord. Furthermore, ELISA demonstrated the high level of IL-17 in the spinal cord following SNL did not come from the peripheral serum. These results suggested IL-17 was produced by Th17 cells. However, a previous study suggested spinal cord-infiltrating $\mathrm{CD}^{+}{ }^{+} \mathrm{T}$ cells are Th1 cells (32). The inconsistency between that and the present study may be associated with the differences in species (rats in the present study, as compared with mice) and method of detection. In addition, Th17 helper cells are not the only cells capable of producing IL-17. In the CNS, expression of IL-17 was also demonstrated in astrocytes located in the active areas of MS lesions (23), or in spinal dorsal horn during CFA-induced inflammatory pain (19). To the best of our knowledge, there are no previous studies regarding the expression of CD4 by astrocytes in 
A

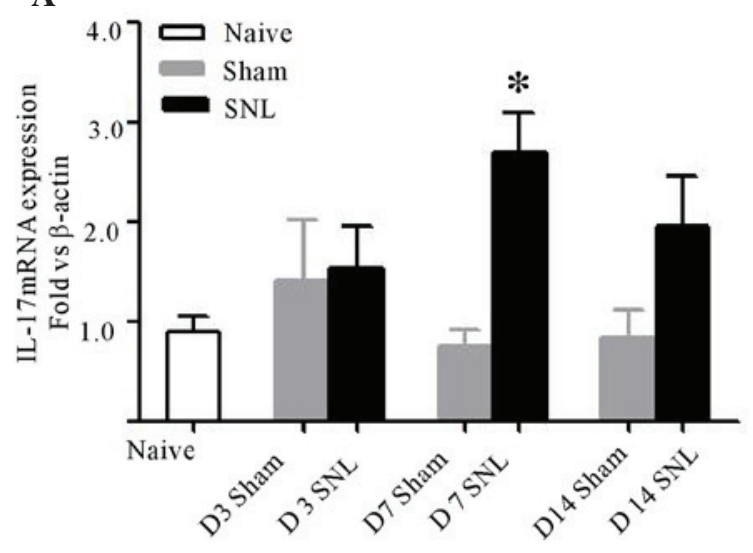

C

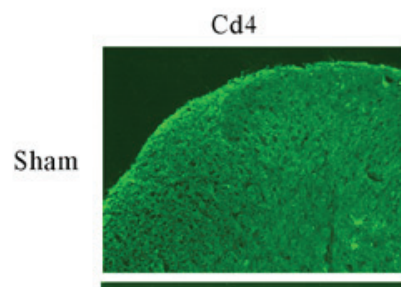

SNL
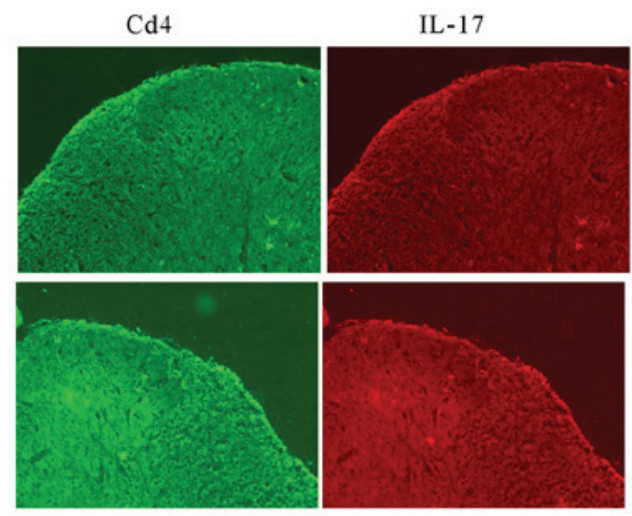

D
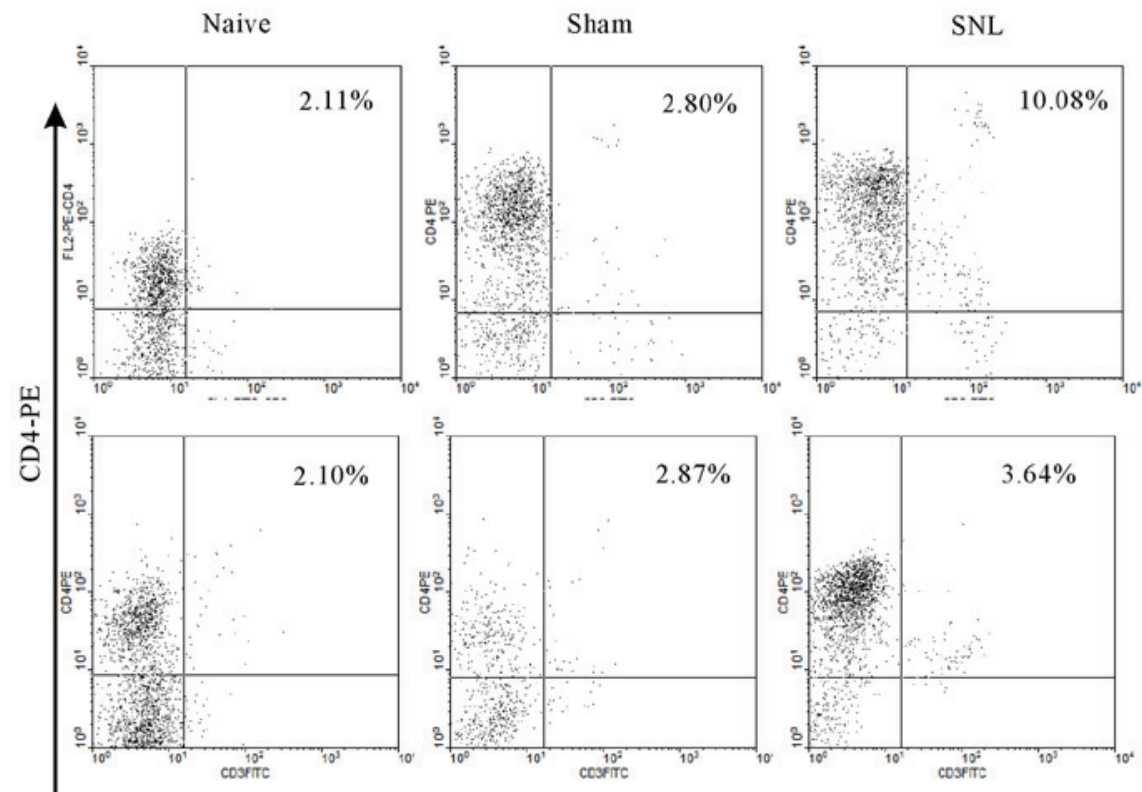

Day 7
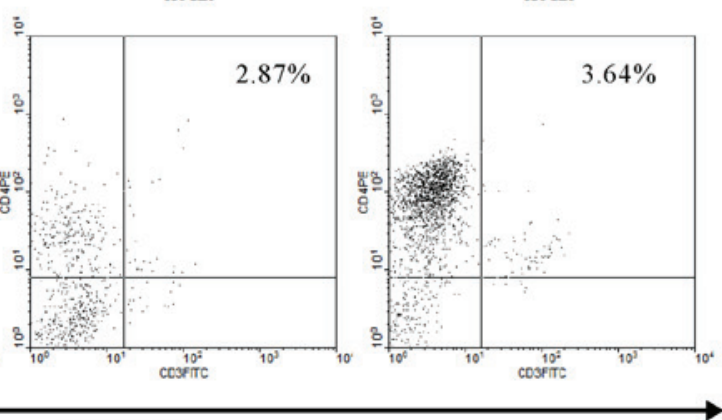

CD3-FITC

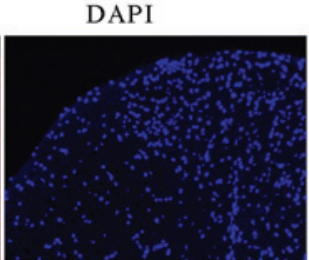

Day 14
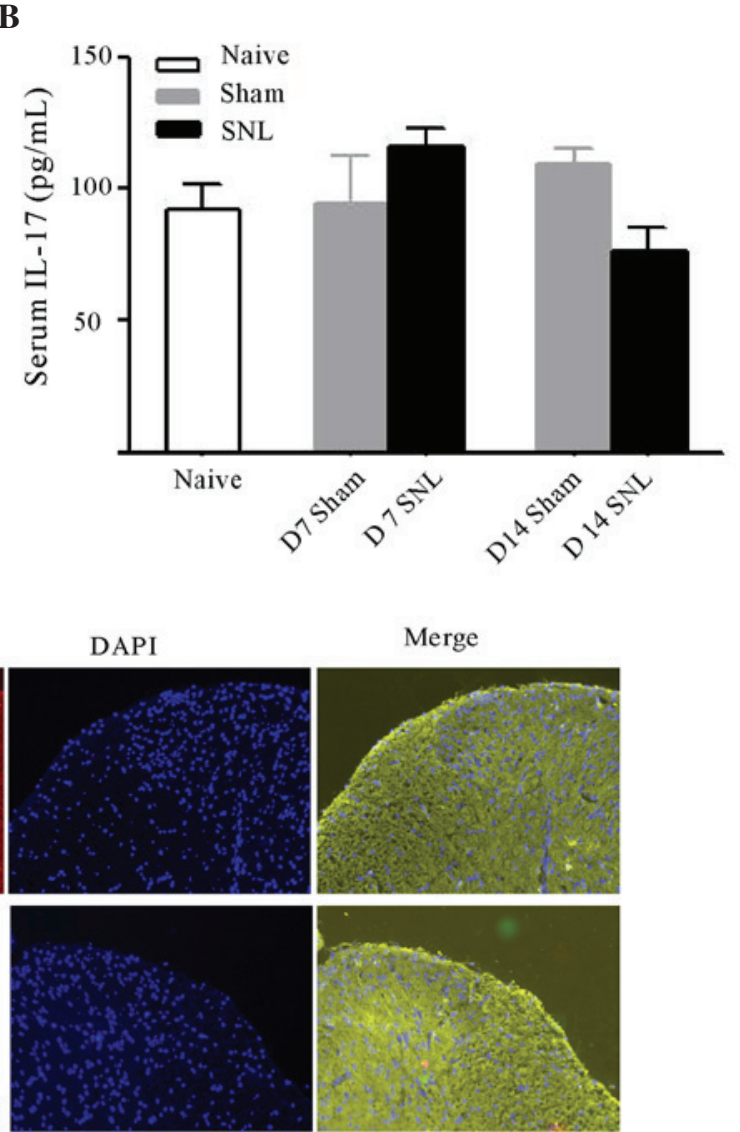

Figure 2. Expression of IL-17 and CD4 in the ipsilateral dorsal horn of L5-6 spinal cord following SNL, and IL-17 in the serum. (A) Quantitative polymerase chain reaction analysis of IL-17 mRNA expression in the rat spinal dorsal horn over time following SNL compared with sham-operated and naive animals, respectively. Data are expressed as mean fold \pm SEM ( $n=4$ /group). " $\mathrm{P}<0.05$ vs. the sham-operated rats at day 7 post-surgery, as determined by two-way analysis of variance, followed by Bonferroni's post-hoc test. (B) Serum IL-17 measured by ELISA at days 7 and 14 post-surgery. Data are expressed as the mean \pm SEM ( $\mathrm{n}=6 /$ group). (C) Double immunofluorescence staining with antibodies against IL-17 and CD4 in the ipsilateral L5 spinal dorsal horn at day 7 following SNL compared with the sham group. The representative images from at least three independent experiments depicted the IL-17A/CD4 overlay, indicating colocalization of IL-17A immunoreactivity with $\mathrm{CD}^{+}$cells. The images are representative images of at least three independent experiments. (D) $\mathrm{CD} 4^{+} \mathrm{T}$ cell infiltration into the rats' ipsilateral lumbar spinal cord at L5-L6 at days 7 and 14 post-SNL, the sham operation or naive rats via FACS. Lumbar spinal cord mononuclear cells were isolated and labeled with anti-rat CD3-FITC and CD4-PE monoclonal antibodies at days 7 and 14, and then were analyzed in CD3 vs. CD4 dot plots. The images are representative of three independent experiments at day 7 and 14, respectively. Each FACS sample consisted of three animals. Percentages of $\mathrm{CD}^{\text {hi }} \mathrm{CD} 4{ }^{\text {hi }}$ population within the total mononuclear cells were analyzed. SNL, spinal nerve ligation; SEM, standard error of the mean; CD, cluster of differentiation; IL, interleukin; FITC, fluorescein isothiocyanate; PE, phycoerythrin; FACS, fluorescence-activated cell sorting; D3, day 3; D7, day 7; D14, day 14; L, lumbar. 
A

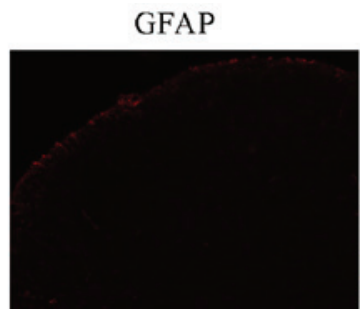

DAPI
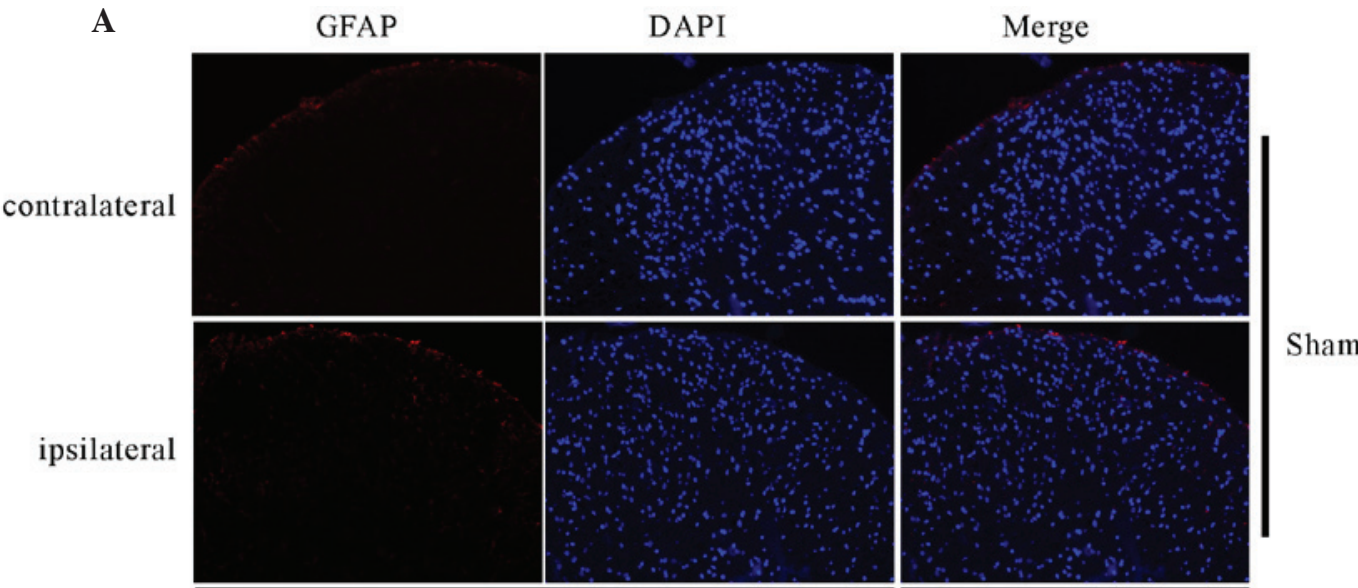

Sham
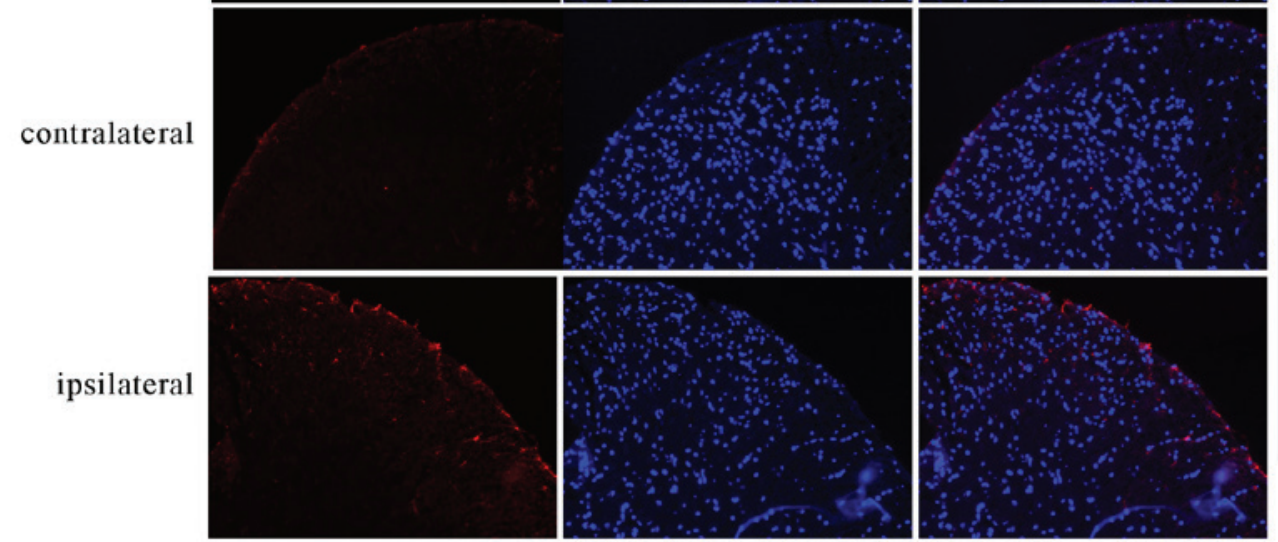

SNL

B
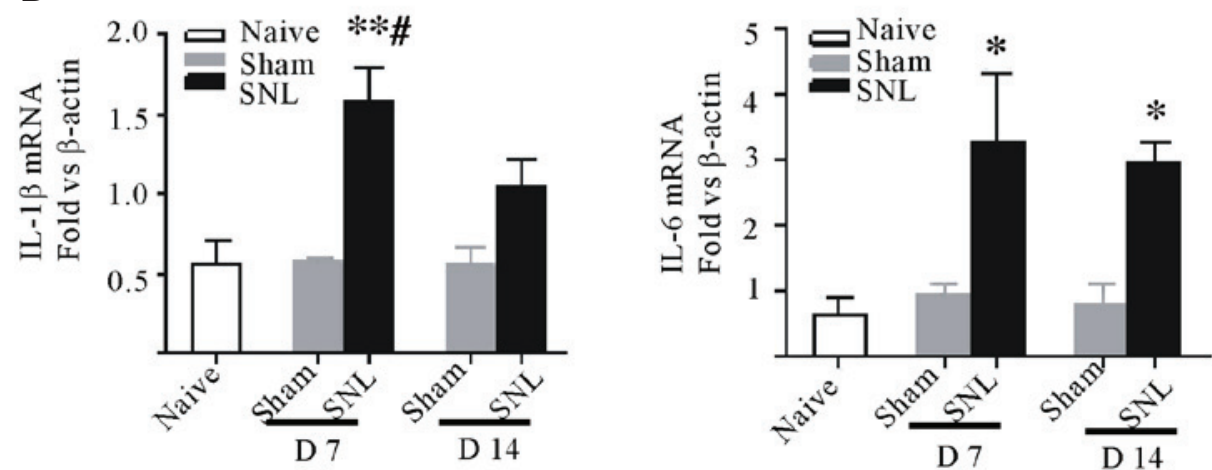

Figure 3. Astrocytic responses and the expression of proinflammatory cytokines in L5-L6 spinal cord following L5 and L6 nerve ligation, sham surgery or naive rats. (A) Fluorescent immunohistochemical staining for GFAP was performed on day 7 post-SNL or sham surgery. GFAP expression markedly increased in the ipsilateral dorsal horn following nerve ligation compared with contralateral and sham-operated rats. Representative photomicrographs of the ipsilateral dorsal horn of L5 lumbar spinal cord are presented (magnification, x20). (B) Quantitative polymerase chain reaction analysis was used to determine the expression of IL-1 $\beta$, IL-6, tumor necrosis factor- $\alpha$ mRNA of the ipsilateral L5-L6 spinal dorsal horn at day 7 and 14 in the rats post-SNL compared with the sham-operated or naive rats. Data are expressed as the mean fold \pm standard error of the mean $(\mathrm{n}=4 / \mathrm{group})$. ${ }^{*} \mathrm{P}<0.05$, ${ }^{* *} \mathrm{P}<0.01 \mathrm{vs}$. the sham-operated/naive rats; ${ }^{\text {P }}<0.05$ vs. the SNL rats at day 14 . GFAP, glial fibrillary acidic protein; SNL, spinal nerve ligation; IL, interleukin; D7, day 7; D14, day 14; L, lumbar.

central neuroinflammation or post nerve injury. Notably, a previous study reports damage-associated molecular patterns activate Toll-like receptor 2 (TLR2) on microglia, which upregulate interleukin-23 expression and subsequent IL-17 induction following cerebral ischemia-reperfusion injury (33). Peripheral nerve injury may lead to microglia activation via TLR2 (34), however, whether or not microglial cells are the source of IL-17 requires further investigation.

Numerous experimental studies suggest that IL-17 is important in a wide range of inflammatory diseases, including autoimmune diseases, such as MS, and other nonimmune neuroinflammatory processes, such as stroke and cerebral ischemia-reperfusion injury (15-18,35). However, its tissue-specific mechanism remains unclear. A previous study demonstrated recombination-activating gene $1 \mathrm{KO}$ mice lacking functional IL-17-producing $\mathrm{T}$ lymphocytes exhibited reduced thermal hyperalgesia due to impaired recruitment of macrophages and expression of macrophage chemoattractant protein in the sciatic nerve following chronic constriction injury, which provided initial evidence of the involvement of IL-17 in neuropathic pain (36). In addition, a previous study reported IL-17 KO mice presented with reduced mechanical allodynia and decreased 
A
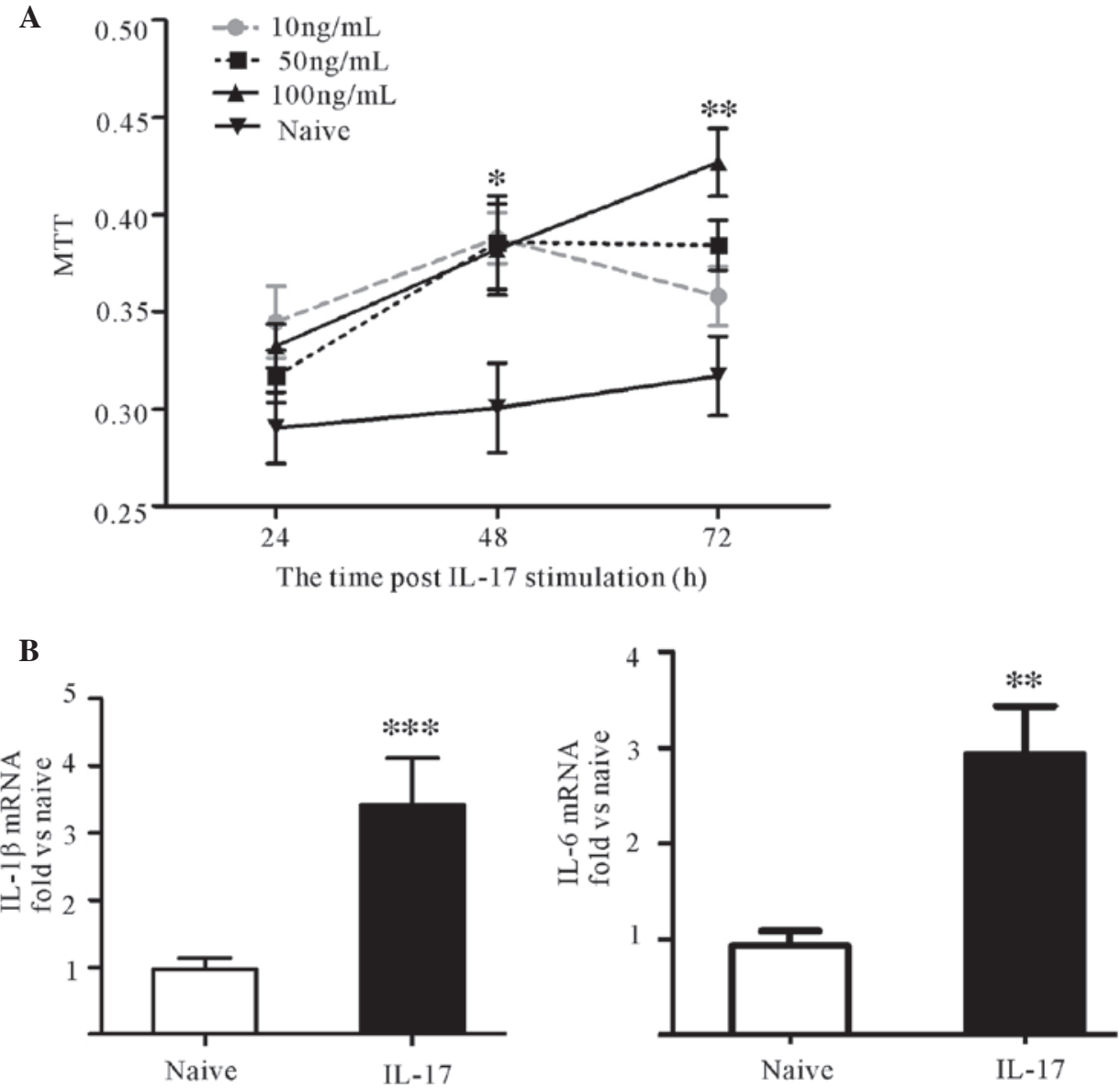

Figure 4. Astrocytic response in vitro treated by IL-17 at different concentrations. (A) MTT was used to detect the proliferation of astrocytes at 24,48 and $72 \mathrm{~h}$ followed by treatment with 10,50 and $100 \mathrm{ng} / \mathrm{ml} \mathrm{IL}-17$. ${ }^{*} \mathrm{P}<0.05,{ }^{* *} \mathrm{P}<0.01$ vs. the naive group. (B) Reverse transcription-quantitative polymerase chain reaction measured the levels of IL-1 $\beta$ and IL-6 in astrocytes stimulated with or without $100 \mathrm{ng} / \mathrm{ml}$ IL-17 for $72 \mathrm{~h} .{ }^{* * *} \mathrm{P}<0.01$, ,*** $\mathrm{P}<0.001$ vs. the naive group. IL, interleukin.

activation of the microglia and astrocytes in the ipisilateral dorsal horn of the spinal cord following partial sciatic nerve ligation indicates IL-17 contributes to neuropathic pain likely via glial cells (2). The results of the immunostaining in the present study indicated that the majority of IL-17 is located in the superficial zone of the ipisilateral spinal dorsal horn, which is also the region of notable astrocytic activation. It is well known that astrocytes constitutively express the receptor of IL-17A (37). Thus, it is reasonable to suggest that IL-17 activates local astrocytes and induces glial response, such as production of proinflammatory cytokines. This possibility is supported by in vitro results from the present study, which demonstrate, in addition to proliferation, stimulation of resting astrocytes by IL-17 increases expression of IL-1 $\beta$ and IL-6, which also occurs in vivo. These mediators lead to central sensitization and pain hypersensitivity $(29,38,39)$. The finding that IL-17 stimulates astrocytes to produce proinflammatory mediators is also demonstrated in MS, experimental autoimmune encephalomyelitis and ischemic brain injury $(12,40)$. However, activation of spinal astrocytes subsequent to nerve injury was not entirely explained by IL-17 and IL-6 expression remained high at day 14 post-SNL and astroglia activation persisted for longer than seven days $(7,9,10)$, which suggests that IL-17-mediated signaling, although important, is not the only mechanism underlying astroglia activation and that there may be independent and/or cooperative mechanisms involving other signals. In addition, Meng et al (20) reported that spinal IL-17 facilitated inflammatory pain by enhancing phosphorylated-NR1 of the N-methyl-D-aspartate receptor in CFA-injected rats. Furthermore, IL-17 can stimulate microglia to upregulate the production of proinflammatory mediators, such as CXCL2, IL-6, and neurotrophic factors (41), and increase the permeability of $\mathrm{BBB}$ in vitro to promote infiltration of proinflammatory cytokines and $\mathrm{CD}^{+} \mathrm{T}$ cells in the CNS. According to these findings, it is possible that other mechanisms may be involved in spinal IL-17-mediated neuropathic pain.

In conclusion, the present study demonstrated that IL-17 is involved in maintaining neuropathic pain, due to activation of astrocytes and the secretion of proinflammatory cytokines. Elevated IL-17 may be produced by infiltrated CD4 ${ }^{+} \mathrm{T}$ cells. However, previous research on IL-17-mediated disorders in the CNS demonstrated cross-interaction between IL-17 and other factors is likely to be involved in the pathogenesis of neuropathic pain. Future studies are required to further investigate and design novel therapies for patients with neuropathic pain.

\section{Acknowledgements}

The present study was supported by grants from the National Natural Science Foundation of China (grant nos. 81370084, 31201346 and 81502663). 


\section{References}

1. Geber C, Baumgärtner U, Schwab R, Müller H, Stoeter P, Dieterich M, Sommer C, Birklein F and Treede RD: Revised definition of neuropathic pain and its grading system: An open case series illustrating its use in clinical practice. Am J Med 122 (Supp 10): S3-S12, 2009.

2. Kim CF and Moalem-Taylor G: Interleukin-17 contributes to neuroinflammation and neuropathic pain following periphera nerve injury in mice. J Pain 12: 370-383, 2011.

3. Saadé NE and Jabbur SJ: Nociceptive behavior in animal models for peripheral neuropathy: Spinal and supraspinal mechanisms. Prog Neurobiol 86: 22-47, 2008.

4. Ellis A and Bennett DL: Neuroinflammation and the generation of neuropathic pain. Br J Anaesth 111: 26-37, 2013.

5. Ramesh G: Novel Therapeutic Targets in Neuroinflammation and Neuropathic Pain. Inflamm Cell Signal 1: pii:e111, 2014

6. Liu F and Yuan H: Role of glia in neuropathic pain. Front Biosci (Landmark Ed) 19: 798-807, 2014.

7. Chiang CY, Wang J, Xie YF, Zhang S, Hu JW, Dostrovsky JO and Sessle BJ: Astroglial glutamate-glutamine shuttle is involved in central sensitization of nociceptive neurons in rat medullary dorsal horn. J Neurosci 27: 9068-9076, 2007.

8. Liu L, Rudin M and Kozlova EN: Glial cell proliferation in the spinal cord after dorsal rhizotomy or sciatic nerve transection in the adult rat. Exp Brain Res 131: 64-73, 2000.

9. Chiang CY, Sessle BJ and Dostrovsky JO: Role of astrocytes in pain. Neurochem Res 37: 2419-2431, 2012.

10. Gao YJ and Ji RR: Targeting astrocyte signaling for chronic pain. Neurotherapeutics 7: 482-493, 2010.

11. Kimelberg HK and Nedergaard M: Functions of astrocytes and their potential as therapeutic targets. Neurotherapeutics 7 : 338-353, 2010

12. Kang Z, Altuntas CZ, Gulen MF, Liu C, Giltiay N, Qin H, Liu L, Qian W, Ransohoff RM, Bergmann C, et al: Astrocyte-restricted ablation of interleukin-17-induced Act1-mediated signaling ameliorates autoimmune encephalomyelitis. Immunity 32 : 414-425, 2010

13. Lin YC, Huang SY, Jean YH, Chen WF, Sung CS, Kao ES, Wang HM, Chakraborty C, Duh CY and Wen ZH: Intrathecal lemnalol, a natural marine compound obtained from Formosan soft coral, attenuates nociceptive responses and the activity of spinal glial cells in neuropathic rats. Behav Pharmacol 22: 739-750, 2011.

14. Tsuda M,Kohro Y, Yano T, Tsujikawa T,Kitano J, Tozaki-Saitoh H, Koyanagi S, Ohdo S, Ji RR, Salter MW and Inoue K: JAK-STAT3 pathway regulates spinal astrocyte proliferation and neuropathic pain maintenance in rats. Brain 134: 1127-1139, 2011.

15. Isailovic N, Daigo K, Mantovani A and Selmi C: Interleukin-17 and innate immunity in infections and chronic inflammation. J Autoimmun 60: 1-11, 2015

16. Lubberts E: IL-17/Th17 targeting: On the road to prevent chronic destructive arthritis? Cytokine 41: 84-91, 2008.

17. Noma N, Khan J, Chen IF, Markman S, Benoliel R, Hadlaq E, Imamura $\mathrm{Y}$ and Eliav E: Interleukin-17 levels in rat models of nerve damage and neuropathic pain. Neurosci Lett 493: 86-91, 2011.

18. Day YJ, Liou JT, Lee CM, Lin YC, Mao CC, Chou AH, Liao CC and Lee HC: Lack of interleukin-17 leads to a modulated micro-environment and amelioration of mechanical hypersensitivity after peripheral nerve injury in mice. Pain 155: 1293-1302, 2014.

19. Zheng H, Zhang Z, Luo N, Liu Y, Chen Q and Yan H: Increased Th17 cells and IL17 in rats with traumatic optic neuropathy. Mol Med Rep 10: 1954-1958, 2014

20. Meng X, Zhang Y, Lao L, Saito R, Li A, Bäckman CM Berman BM, Ren K, Wei PK and Zhang RX: Spinal interleukin-17 promotes thermal hyperalgesia and NMDA NR1 phosphorylation in an inflammatory pain rat model. Pain 154 294-305, 2013

21. Cao L and DeLeo JA: CNS-infiltrating CD4+ T lymphocytes contribute to murine spinal nerve transection-induced neuropathic pain. Eur J Immunol 38: 448-458, 2008.
22. Costigan M, Moss A, Latremoliere A, Johnston C, Verma-Gandhu M, Herbert TA, Barrett L, Brenner GJ, Vardeh D, Woolf CJ and Fitzgerald M: T-cell infiltration and signaling in the adult dorsal spinal cord is a major contributor to neuropathic pain-like hypersensitivity. J Neurosci 29 14415-14422, 2009.

23. Olechowski CJ, Truong JJ and Kerr BJ: Neuropathic pain behaviours in a chronic-relapsing model of experimental autoimmune encephalomyelitis (EAE). Pain 141: 156-164, 2009.

24. Kim SH and Chung JM: An experimental model for peripheral neuropathy produced by segmental spinal nerve ligation in the rat. Pain 50: 355-363, 1992.

25. Chaplan SR, Bach FW, Pogrel JW, Chung JM and Yaksh TL: Quantitative assessment of tactile allodynia in the rat paw. J Neurosci Methods 53: 55-63, 1994.

26. Livak KJ and Schmittgen TD: Analysis of relative gene expression data using real-time quantitative PCR and the 2(-Delta Delta C(T)) method. Methods 25: 402-408, 2001.

27. Beeton $\mathrm{C}$ and Chandy KG: Isolation of mononuclear cells from the central nervous system of rats with EAE. J Vis Exp: 527 , 2007.

28. Kerstetter AE and Miller RH: Isolation and culture of spinal cord astrocytes. Methods Mol Biol 814: 93-104, 2012.

29. Clark AK, Old EA and Malcangio M: Neuropathic pain and cytokines: Current perspectives. J Pain Res 6: 803-814, 2013.

30. Iwakura Y, Ishigame H, Saijo S and Nakae S: Functional specialization of interleukin-17 family members. Immunity 34: 149-162, 2011.

31. Hu P, Bembrick AL, Keay KA and McLachlan EM: Immune cell involvement in dorsal root ganglia and spinal cord after chronic constriction or transection of the rat sciatic nerve. Brain Behav Immun 21: 599-616, 2007.

32. Draleau K, Maddula S, Slaiby A, Nutile-McMenemy N, De Leo J and Cao L: Phenotypic identification of spinal cord-infiltrating CD4 T lymphocytes in a murine model of neuropathic pain. J Pain Relief (Suppl 3): 003, 2014.

33. Zhang J, Takahashi HK, Liu K, Wake H, Liu R, Maruo T, Date I, Yoshino T, Ohtsuka A, Mori S and Nishibori M: Anti-high mobility group box-1 monoclonal antibody protects the blood-brain barrier from ischemia-induced disruption in rats. Stroke 42: 1420-1428, 2011.

34. Stokes JA, Cheung J, Eddinger K, Corr M and Yaksh TL: Toll-like receptor signaling adapter proteins govern spread of neuropathic pain and recovery following nerve injury in male mice. J Neuroinflammation 10: 148, 2013

35. Knier B, Rothhammer V, Heink S, Puk O, Graw J, Hemmer B and Korn T: Neutralizing IL-17 protects the optic nerve from autoimmune pathology and prevents retinal nerve fiber layer atrophy during experimental autoimmune encephalomyelitis. J Autoimmun 56: 34-44, 2015.

36. Kleinschnitz C, Hofstetter HH, Meuth SG, Braeuninger S, Sommer C and Stoll G: T cell infiltration after chronic constriction injury of mouse sciatic nerve is associated with interleukin-17 expression. Exp Neurol 200: 480-485, 2006.

37. Xu S and Cao X: Interleukin-17 and its expanding biological functions. Cell Mol Immunol 7: 164-174, 2010.

38. Kawasaki Y, Zhang L, Cheng JK and Ji RR: Cytokine mechanisms of central sensitization: Distinct and overlapping role of interleukin-1beta, interleukin-6 and tumor necrosis factor-alpha in regulating synaptic and neuronal activity in the superficial spinal cord. J Neurosci. 28: 5189-5194, 2008.

39. Guo W, Wang H, Watanabe M, Shimizu K, Zou S, LaGraize SC, Wei F, Dubner R and Ren K: Glial-cytokine-neuronal interactions underlying the mechanisms of persistent pain. J Neurosci 27: 6006-6018, 2007.

40. Gelderblom M, Weymar A, Bernreuther C, Velden J, Arunachalam P, Steinbach K, Orthey E, Arumugam TV, Leypoldt F, Simova O, et al: Neutralization of the IL-17 axis diminishes neutrophil invasion and protects from ischemic stroke. Blood 120: 3793-3802, 2012.

41. Kawanokuchi J, Shimizu K, Nitta A, Yamada K, Mizuno T, Takeuchi $\mathrm{H}$ and Suzumura A: Production and functions of IL-17 in microglia. J Neuroimmunol 194: 54-61, 2008. 